\title{
Computational techniques for assessing the reliability and sustainability of electrical power systems: a review
}

\begin{abstract}
Power systems employ measures of reliability indices to indicate the effectiveness a power system to perform its basic function of supplying electrical energy to its consumers. The amount of energy required in a generating system to ensure an adequate supply of electricity is determined using analytical and simulation techniques. This study focuses on reviewing the generation reliability assessment methods of power systems using Monte Carlo simulation (MCS) and variance reduction techniques (VRTs). MCS is a very flexible method for reliability assessment of the power systems, by the sequential process it can imitate the random nature of the system components and can be broadly classified into two, sequential and non-sequential simulations. A brief introduction to MCS is provided. Unlike analytical methods, MCS can be used to quantitatively estimate the system reliability in even the most complex system generating capacity situations. The major drawback of the MCS is that it requires more computational time to reach for converging with estimated the values of reliability indices. This paper presents an effective methods for accelerating MCS in power system reliability assessment. VRT used is to manipulate the way each sample of an MCS is defined in order to both preserve the randomness of the method and decrease the variance of the estimation. In addition, the study presents detailed descriptions of generation reliability assessment methods, in order to provide computationally efficient and precise methodologies based on the pattern simulation technique. These methodologies offer significantly improved computational ability during evaluations of power generation reliability.
\end{abstract}

Keyword: Monte Carlo simulation techniques; Variance reduction techniques; Adequacy assessment methods; Latin hypercube sampling; Cross-entropy 